\title{
Improved Short-Term Outcomes of Osteochondral Lesions of the Knee Following Arthroscopic Treatment With Bone Marrow Aspirate Concentrate and Cartilage-Derived Matrix
}

\author{
Iciar M. Dávila Castrodad, M.D., Erica S. Simone, M.S., A.T.C., O.T.C., \\ Jennifer Kurowicki, M.D., Justin X. Melendez, B.S., Samuel J. Mease, M.D., \\ Vincent K. McInerney, M.D., and Anthony J. Scillia, M.D.
}

\begin{abstract}
Purpose: To assess the postoperative objective, subjective, and functional outcomes as well as complication rates in osteochondral defect patients treated with bone marrow aspirate concentrate (BMAC) and cartilage-derived matrix (CDM) during knee arthroscopy. Methods: A retrospective chart review was performed for patients treated arthroscopically with BMAC and CDM between August 2015 and August 2018 and had more than 1-year follow-up. Demographic factors such as age, sex, body mass index, and comorbidities were collected for all patients. Size and location of the osteochondral lesions also were documented. Results: A total of 14 patients were identified with a mean follow-up of 19 months. On average, patients were 34 years of age (range $16-58$ years) and $43 \%$ were female. Postoperatively, knee flexion increased by $8^{\circ}$ from $124^{\circ}$ to $132^{\circ}(P=.002)$. All patients regained full extension; however, 1 patient later acquired a $2^{\circ}$ extension contracture after a traumatic event. The average hamstring strength significantly increased from 4.1 to 4.6 postoperatively $(P=.33)$. The average quadriceps strength significantly increased from 4.0 to 4.5 postoperatively $(P=$ $.007)$. Mean visual analog scale scores significantly decreased postoperatively $(4.5 \mathrm{vs} 1.4 ; P=.001)$. There was a significant increase in Knee Outcome Survey Activities of Daily Living scores (53.8 vs 92.9; $P=.007$ ). Mean Knee Outcome SurveySports scores also increased, although this was nonsignificant $(28.2$ vs $79.5 ; P=.560)$. No significant differences were noted in pain and functional outcomes when stratified by the osteochondral defect size and location. Complications included a stitch abscess, Baker's cyst, and residual pain treated with hyaluronic acid injection. Conclusions: This study demonstrated arthroscopic BMAC and CDM implantation appears to be safe and has the potential to improve patient outcomes in the short-term postoperative period. Level of Evidence: IV, therapeutic case series.
\end{abstract}

$\mathbf{O}$ steochondral defects of the knee are identified during more than $60 \%$ of arthroscopic procedures. ${ }^{1-3}$ Due to their limited healing capacity, these lesions remain a challenge to definitively treat. ${ }^{4-6}$ Given their heterogeneity, several procedures have been formulated to treat symptomatic patients with

From the Department of Orthopaedic Surgery, St. Joseph's University Medical Center, Paterson (I.M.D.C., J.K., S.J.M., V.K.M., A.J.S.); New Jersey Orthopaedic Institute, Wayne (I.M.D.C., E.S.S., J.X.M., V.K.M., A.J.S.); and Department of Orthopaedic Surgery, Hackensack Meridian School of Medicine at Seton Hall University, Nutley (V.K.M., A.J.S.), New Jersey, U.S.A.

The authors report the following potential conflicts of interest or sources of funding: E.S.S reports other from Johnson $\theta$ Johnson and Merck, outside the submitted work. V.K.M. reports other from Topical Gear, MD Advantage Board of Trustees, and from New Jersey Orthopedic Society, outside the submitted work. A.J.S. reports other from ISO-Biologics, personal fees from Mitek, and other from New Jersey Orthopedic Institute, outside the submitted work. Full ICMJE author disclosure forms are available for this article online, as supplementary material. osteochondral defects based on factors such as defect size, lesion depth, patient age, and concomitant intraarticular pathology. ${ }^{7,8}$ These procedures include microfracture (MF), juvenile articular cartilage implantation, osteochondral autograft, or allograft, transfer system (OATs), autologous chondrocyte

Received January 13, 2020; accepted November 3, 2020

Address correspondence to Anthony J. Scillia, M.D., St. Joseph's University Medical Center, Hackensack Meridian School of Medicine at Seton Hall University, Department of Orthopaedic Surgery, 703 Main St., Paterson, NJ 07503. E-mail: anthonyjscillia@gmail.com

(C) 2021 THE AUTHORS. Published by Elsevier Inc. on behalf of the Arthroscopy Association of North America. This is an open access article under the CC BY-NC-ND license (http://creativecommons.org/licenses/by-nc-nd/4.0/). 2666-061X/2049

https://doi.org/10.1016/j.asmr.2020.11.002 
implantation (ACI), and matrix-induced ACI and are generally indicated based on lesion type. ${ }^{9,10}$ Despite these numerous options, long-term outcomes have shown varied results and the optimal treatment for these defects remains unclear. ${ }^{11}$ Thus, newer therapies including biologic and scaffold-based treatments have emerged with efforts to improve postoperative outcomes in patients with focal osteochondral lesions.

Among the biologic therapies, cell-based injections including bone marrow aspirate concentrate (BMAC) have been proposed and applied in clinical practice. Bone marrow-derived mesenchymal stem cells (MSCs) have a theoretical advantage over MSCs from other origins in that they have a greater differentiation capacity and thus are believed to aid in tissue regeneration. ${ }^{12}$ Following treatment with BMACs, preclinical studies have demonstrated an increased fill of cartilage defects and well-integrated repair tissue with greater type-II collagen content. ${ }^{13,14}$ Scaffold-based cartilage treatments are another method used in osteochondral defect treatment that also have reached clinical practice. These scaffolds are available as synthetic matrices or as natural materials, including collagen derivates and hyaluronic acid (HA). ${ }^{15}$ While cell-based constructs are more commonly studied and yield promising outcomes, cell-free scaffolds also have demonstrated favorable outcomes in the short-term. ${ }^{16-18}$

Nonetheless, cartilage-derived matrix (CDM) scaffolds have recently gained traction because, in contrast to marrow-stimulating procedures such as MF, the repair tissue has been shown to have better long-term viability. ${ }^{19}$ Current osteochondral defect treatments are associated with several drawbacks. MF has questionable durability with poor outcomes beyond the short term and is correlated with low rates of return to play in high level athletes. ${ }^{5,20,21}$ The OATs procedure is associated with donor-site morbidity, ACI is costly as it requires multiple operations, and with the exception of $\mathrm{MF}$, these procedures cannot be performed through a minimally invasive approach. ${ }^{22-24}$ Hence, BMAC and CDM scaffolds may offer a promising alternative.

In recent years, several new developments have emerged to address osteochondral lesions. Thus, it is important to evaluate the postoperative outcomes among patients who undergo newer biologic and scaffold-based treatments. Therefore, the purpose of this study was to assess the postoperative objective, subjective, and functional outcomes as well as complication rates in osteochondral defect patients treated with BMAC and CDM during knee arthroscopy. Specifically, we assessed the postoperative objective, subjective, and functional outcomes as well as complication rates in patients treated with BMAC and CDM during knee arthroscopy. We hypothesized that osteochondral defect patients treated arthroscopically with BMAC and
CDM would have improved postoperative outcomes and no associated adverse events.

\section{Methods}

\section{Patient Selection}

Following institutional review board approval, we performed a retrospective analysis on patients diagnosed with osteochondral defects of the knee. Patients were included if they were arthroscopically treated with BMAC and CDM implantation by a single, sportsmedicine fellowship-trained orthopaedic surgeon at a single, academic institution between August 2015 and August 2018 and had more than 1-year follow-up. Patients with a diagnosis of knee osteoarthritis, based on radiographic analysis (less than $2 \mathrm{~mm}$ of joint space on weight-bearing radiographs), or inflammatory arthropathy, who received other forms of treatment, or lacked 1 year of follow-up were excluded. Demographic factors such as age, sex, body mass index, and comorbidities were collected for all patients. Although subchondral one depth was not recorded, other characteristics of the osteochondral lesion obtained during chart review such as size and location also were documented for all patients.

\section{Surgical Indications and Treatment}

Patients who presented with knee pain, swelling, mechanical symptoms, limited motion, and functional capabilities were diagnosed with an osteochondral defect lesion and those who did not respond to conservative measures including physical therapy, nonsteroidal inflammatory drug use, and intra-articular injections were indicated for and underwent arthroscopic treatment with BMAC and CDM.

Surgery consisted of bone marrow aspiration and centrifugation (Magellan ISTO Biologics, Hopkinton, MA), defect preparation, BMAC and CDM (BioCartilage; Arthrex, Naples, FL) implantation, and a final intra-articular BMAC injection. A total of $60 \mathrm{~mL}$ of bone marrow were aspirated using a Jamshidi needle 3 $\mathrm{cm}$ posterior to the anterior superior iliac spine. During aspiration, the syringe was rotated every $5 \mathrm{~mL}$ until the total amount was obtained. In sterile fashion, the suspension was concentrated to $7 \mathrm{~mL}$ via centrifugation. The amount of MSCs was not evaluated for each patient. One milliliter of BMAC was combined with the cartilage-derived scaffold while the remaining $6 \mathrm{~mL}$ were set aside for the intra-articular injection. Before the matrix implantation, diagnostic arthroscopy was performed to identify the presence of and address concomitant pathology. Examples of concomitant procedures included loose body removal, revision anterior cruciate ligament reconstruction, distal femoral osteotomy, revision medial patellofemoral ligament 
reconstruction, lateral release, and partial meniscectomy (Table 1). When present, pathologic patellofemoral or tibiofemoral malalignment was addressed with an osteotomy. Other concomitant procedures were performed when indicated, including the associated presence of preoperative subchondral insufficiency fracture adjacent to the lesion with percutaneous skeletal fixation with calcium phosphate cement (Zimmer subchondroplasty). The osteochondral defect was then identified and debrided to healthy bleeding subchondral bone and a contained rim. The fluid was removed and the lesion was thoroughly dried and grafted with the mixture of $1 \mathrm{~mL}$ of BMAC and CDM and covered with fibrin glue. Lastly, the remaining 6 $\mathrm{mL}$ of BMAC was injected intra-articularly once the fibrin glue solidified. A full description of the technique has been previously defined. ${ }^{25}$ Standard perioperative care included 1 preoperative dose of intravenous antibiotics and postoperative thromboembolic prophylaxis with $325 \mathrm{mg}$ of aspirin twice a day. Postoperatively, patients with tibiofemoral lesions were $50 \%$ partial weight-bearing for 4 to 6 weeks without a brace while patients with patellofemoral lesions were allowed weight-bearing as tolerated with a hinged knee brace

Table 1. Demographics for Osteochondral Defect Patients Treated With BMAC and CDM

\begin{tabular}{|c|c|}
\hline Demographics of the Patients, $\mathrm{N}=14$ & $\mathrm{n}(\%)$ \\
\hline \multicolumn{2}{|l|}{ Sex } \\
\hline Female & $6(42.9)$ \\
\hline Male & $8(57.1)$ \\
\hline Age, y & $34(16-58)$ \\
\hline \multicolumn{2}{|l|}{ Number of comorbidities } \\
\hline 0 & $7(50)$ \\
\hline 1 & $3(21.4)$ \\
\hline 2 & $4(28.6)$ \\
\hline BMI & $27.7(21.8-37.2)$ \\
\hline Size of lesion, $\mathrm{cm}^{2}$ & $1.8(.7-3.0)$ \\
\hline \multicolumn{2}{|l|}{ Location of lesion } \\
\hline Patella & $5(35.7)$ \\
\hline Lateral femoral condyle & $5(35.7)$ \\
\hline Medial femoral condyle & $4(28.6)$ \\
\hline Trochlea & $2(14.2)$ \\
\hline \multicolumn{2}{|l|}{ Concomitant procedures } \\
\hline Loose body removal & $5(35.7)$ \\
\hline Revision ACL reconstruction & $2(14.2)$ \\
\hline Distal femoral osteotomy & $1(7.1)$ \\
\hline Revision MPFL reconstruction & $1(7.1)$ \\
\hline Lateral release & $3(21.9)$ \\
\hline Partial meniscectomy & $4(28.6)$ \\
\hline Plica excision & $2(14.2)$ \\
\hline Tibial tubercle osteotomy & $1(7.1)$ \\
\hline Chondroplasty & $2(14.2)$ \\
\hline Subchondroplasty & $3(21.9)$ \\
\hline Follow-up, mo & $19(12-31)$ \\
\hline
\end{tabular}

locked in extension for 4 to 6 weeks while weightbearing. All patients were allowed passive range of motion (ROM) as tolerated with isometric strengthening for 6 weeks followed by a progressive strengthening program. If patients continued with symptoms at 5 months, a series of intra-articular HA injections were offered postoperatively. Postoperative magnetic resonance imaging was not performed on a routine basis.

\section{Variables}

Physical examination findings included ROM (i.e., flexion and extension) and hamstring and quadriceps strength. Passive knee flexion and extension were assessed with the patient in the supine position while manual muscle strength testing, also performed in the supine position, was based on a standard grading scale of 0 to 5 . Pain intensity was measured by using visual analog scale (VAS) scores. Functional outcomes were assessed with the University of California Los Angeles Activity Score (UCLA), Knee Outcome Survey Activities of Daily Living (KOS-ADL), and KOS-Sports Scores. Pain and functional outcomes were further stratified based on osteochondral defect size $\left(<2 \mathrm{~cm}^{2}\right.$ vs $>2 \mathrm{~cm}^{2}$ ) and location (patella and trochlea vs medial and lateral femoral condyles).$^{8}$ All objective, subjective, and functional outcomes were assessed at final followup. Complications were documented and were defined as wound complications, implant or hardware-related problems, and subsequent surgeries.

\section{Data Analysis}

Descriptive analyses were performed to assess patient demographics and physical examination findings, which were both reported as frequencies. Paired samples $t$ tests and Student $t$ tests were used to compare ROM, muscle strength, pain, and functional outcome scores. Effect size was calculated for ROM, muscle strength, VAS, UCLA, KOS-ADL, and KOS-Sports scores. Effect sizes were considered small, medium, and large if they measured $0.2,0.5$, and 0.8 , respectively. $\chi^{2}$ tests were performed to compare hamstring and quadriceps strength between patients when stratified by osteochondral defect size and location. A $P$ value of .05 was set as the threshold for statistical significance. All statistical analysis was performed using SPSS, version 22 (IBM Corp, Armonk, NY).

\section{Results}

\section{Patients Included in Final Analysis}

A total of 75 knee osteochondral defect patients were identified. Of these, 21 patients did not receive CDM implantation and 4 patients did not receive BMAC. Twenty-three patients received other modes of treatment and were thus excluded. Of the remaining patients, 13 had follow-up of less than 1 year. Therefore, 
14 patients fit the inclusion criteria and were included for analysis. On average, patients had 19 months of follow-up (range 12-31 months), were 34 years of age (range 16-58 years), and $43 \%$ were female (Table 1). The mean body mass index was 27.7 (range 21.8-37.2). The number of comorbidities among the patients ranged from 0 to 2 . All patients had an International Cartilage Repair Society (ICRS) grade of IV. $^{26}$ The average size of the osteochondral defect lesion was 1.8 $\mathrm{cm}^{2}$ (range $0.7-3 \mathrm{~cm}^{2}$ ). The patella and lateral femoral condyle represented the most common locations for the osteochondral defects, followed by the medial femoral condyle and the trochlea. Twelve patients had one osteochondral lesion, whereas 2 patients were found to have 2 lesions; 1 patient had lesions in the patella and trochlea and the other patient had lesions in the lateral and medial femoral condyles. While all patients had at least 1 concomitant procedure, loose body removal $(35.7 \%)$ and partial meniscectomy $(28.6 \%)$ were the most commonly performed concomitant procedures. Finally, patient-reported outcome measures were completed by $13(93 \%)$ patients.

\section{Physical Examination Findings}

Postoperatively, knee flexion increased by $8^{\circ}$ from $124^{\circ}$ to $132^{\circ}(P=.002)$ (Table 2$)$. All patients regained full extension; however, 1 patient later acquired a $2^{\circ}$ extension contracture after a traumatic event which affected his operated extremity. Preoperative hamstring strength ranged from $4-$ to 5 whereas the postoperative strength ranged from 4 to 5 . The average hamstring strength significantly increased from 4.1 to 4.6 postoperatively $(P=.33)$. Similarly, preoperative quadriceps strength ranged from $3+$ to 5 whereas postoperative strength increased to a range of 4 to 5 . The average quadriceps strength significantly increased from 4.0 to 4.5 postoperatively $(P=.007)$. The effect sizes were large $(>0.80)$ in regards to hamstring strength (0.94) and quadriceps strength (0.96).

\section{Pain and Functional Outcomes}

Mean preoperative VAS scores significantly decreased postoperatively ( 4.5 vs $1.4 ; P=.001)$. There was a slight increase in mean UCLA scores, although this was nonsignificant (5.7 vs $6.6 ; P=.455$ ). There was a significant increase in postoperative KOS-ADL scores (53.8 vs 92.9; $P=.007$ ). Mean KOS-Sports scores also increased, though this was nonsignificant (28.2 vs 79.5; $P=.560$ ). No significant differences were noted in pain and functional outcomes when stratified by osteochondral defect size (Table 3). Similarly, there were no differences in pain and functional outcomes when stratified by osteochondral defect location, with the exception of postoperative UCLA scores (Table 4). Patients with patella and trochlea lesions had lower postoperative UCLA scores than patients with femoral
Table 2. Clinical and Functional Outcomes of Osteochondral Defect Patients Treated With BMAC and CDM

\begin{tabular}{|c|c|c|}
\hline Demographics of the Patients, $\mathrm{N}=14$ & $\mathrm{~N}(\%)$ & $P$ Value \\
\hline \multicolumn{3}{|l|}{ Physical examination findings } \\
\hline Preoperative flexion, ${ }^{\circ}$ & $123.9(3.6)$ & \\
\hline Postoperative flexion, ${ }^{\circ}$ & $131.5(6.3)$ & .002 \\
\hline Effect size & 1.06 & \\
\hline \multicolumn{3}{|l|}{ Hamstring* } \\
\hline \multicolumn{3}{|l|}{ Preoperative strength } \\
\hline $4-$ & $3(33.3)$ & \\
\hline 4 & $5(55.6)$ & \\
\hline 5 & $1(11.1)$ & \\
\hline \multicolumn{3}{|l|}{ Postoperative strength } \\
\hline 4 & $5(45.5)$ & \\
\hline 5 & $6(54.5)$ & \\
\hline Preoperative strength & $4.1(0.35)$ & \\
\hline Postoperative strength & $4.6(0.52)$ & .33 \\
\hline Effect size & 0.94 & \\
\hline \multicolumn{3}{|l|}{ Quadriceps* } \\
\hline \multicolumn{3}{|l|}{ Preoperative strength } \\
\hline $3+$ & $2(15.4)$ & \\
\hline $4-$ & $5(38.5)$ & \\
\hline 4 & $5(38.5)$ & \\
\hline 5 & $1(7.7)$ & \\
\hline \multicolumn{3}{|l|}{ Postoperative strength } \\
\hline 4 & $7(53.8)$ & \\
\hline 5 & $6(46.2)$ & \\
\hline Preoperative strength & $4.0(0.43)$ & \\
\hline Postoperative strength & $4.5(0.52)$ & .007 \\
\hline Effect size & 0.96 & \\
\hline \multicolumn{3}{|l|}{ Pain scores } \\
\hline Preoperative VAS & $4.5(2.4)$ & \\
\hline Postoperative VAS & $1.4(1.6)$ & .001 \\
\hline Effect size & 1.32 & \\
\hline \multicolumn{3}{|l|}{ Functional outcomes } \\
\hline Preoperative UCLA & $5.7(2.4)$ & \\
\hline Postoperative UCLA & $6.6(2.5)$ & .455 \\
\hline Effect size & 0.23 & \\
\hline Preoperative KOS-ADL & $53.8(14.3)$ & \\
\hline Postoperative KOS-ADL & $92.9(12.4)$ & .007 \\
\hline Effect size & 6.76 & \\
\hline Preoperative KOS-Sports & $28.2(15.7)$ & .560 \\
\hline Postoperative KOS-Sports & $79.5(20.3)$ & \\
\hline Effect size & 1.51 & \\
\hline \multicolumn{3}{|c|}{$\begin{array}{l}\text { NOTE. Values are given as the mean and standard deviation in } \\
\text { parentheses. } \\
\text { ADL, activities of daily living; BMAC, bone marrow aspirate } \\
\text { concentrate; CDM, cartilage-derived matrix; KOS, Knee Outcome } \\
\text { Survey; UCLA: University of California, Los Angeles Activity Score; } \\
\text { VAS, visual analog scale. } \\
\text { *Strength is based on a grading scale of } 0-5 \text {. }\end{array}$} \\
\hline
\end{tabular}

condyle lesions (5.5 vs 8.4; $P=.04$ ). The effect sizes were large $(>0.80)$ in regards to knee flexion (1.06), VAS (1.32), KOS-ADL (6.76), and the KOS-Sports (1.51). The effect size for the UCLA score was small $(0.23)$.

\section{Complications}

There was a $29 \%$ complication rate, as 4 postoperative complications were documented (Table 5). One patient had a stitch abscess, which resolved with 
Table 3. Clinical and Functional Outcomes Stratified by Osteochondral Size

\begin{tabular}{|c|c|c|c|}
\hline $\mathrm{N}(\%)$ & $<2 \mathrm{~cm}^{2}$ & $>2 \mathrm{~cm}^{2}$ & $P$ Value \\
\hline \multicolumn{4}{|l|}{ Physical examination findings } \\
\hline Preoperative flexion, ${ }^{\circ}$ & $126.0(4.2)$ & $122(2.7)$ & .11 \\
\hline Postoperative flexion, ${ }^{\circ}$ & $130.8(7.4)$ & $132.0(4.5)$ & .77 \\
\hline \multicolumn{4}{|l|}{ Hamstring } \\
\hline \multicolumn{4}{|l|}{ Preoperative strength } \\
\hline $4-$ & $0(0.0)$ & $3(60.0)$ & \\
\hline 4 & $4(66.7)$ & $0(0.0)$ & .03 \\
\hline \multicolumn{4}{|l|}{ Postoperative strength } \\
\hline 4 & $2(33.3)$ & $1(20.0)$ & \\
\hline 5 & $2(33.3)$ & $3(60.0)$ & .68 \\
\hline \multicolumn{4}{|l|}{ Quadriceps } \\
\hline \multicolumn{4}{|l|}{ Preoperative strength } \\
\hline $3+$ & $0(0.0)$ & $1(20.0)$ & \\
\hline $4-$ & $1(16.7)$ & $4(80.0)$ & \\
\hline 4 & $4(66.7)$ & $0(0.0)$ & .05 \\
\hline \multicolumn{4}{|l|}{ Postoperative strength } \\
\hline 4 & $4(66.7)$ & $1(20.0)$ & \\
\hline 5 & $2(33.3)$ & $3(60.0)$ & .23 \\
\hline \multicolumn{4}{|l|}{ Pain scores } \\
\hline Preoperative VAS & $4.1(3.1)$ & $5.2(2.6)$ & .56 \\
\hline Postoperative VAS & $1.6(1.5)$ & $1.2(2.2)$ & .74 \\
\hline \multicolumn{4}{|l|}{ Functional Outcomes } \\
\hline Preoperative UCLA & $5.6(2.9)$ & $5.0(3.2)$ & .78 \\
\hline Postoperative UCLA & $8.6(1.1)$ & $6.0(3.4)$ & .17 \\
\hline Preoperative KOS-ADL & 38.6 & 55.7 & \\
\hline Postoperative KOS-ADL & $88.9(13.0)$ & $77.1(27.9)$ & .43 \\
\hline Preoperative KOS-Sports & $32.7(20.5)$ & 34.5 & .95 \\
\hline Postoperative KOS-Sports & $80.7(17.9)$ & $74.2(21.7)$ & .62 \\
\hline
\end{tabular}

routine wound care. Another patient developed a Baker's cyst and subsequently underwent an open cyst excision. One patient complained of painful hardware, which consisted of 2 tibial tubercle screws from the tibial tubercle osteotomy that was performed concomitantly with BMAC and CDM implantation. This was performed to address the pathologic patellar malalignment with a tibial tubercle- trochlear groove distance greater than $20 \mathrm{~mm}$. Both screws were later removed. One patient continued with residual symptoms and received a series of $3 \mathrm{HA}$ injections at 5 months postoperatively.

\section{Discussion}

We found that physical examination findings, VAS scores, and KOS- ADL scores significantly improved following BMAC and CDM implantation, with approximately one-third of patients having minor complications and no severe complications. Arthroscopic BMAC and CDM implantation has become one of many treatment options available for osteochondral defects of the knee. Cell-based injections such as BMAC and scaffold-based treatments have demonstrated improvements in cartilage defects in preclinical studies and have now reached clinical practice. Thus, it is important to assess the postoperative outcomes among patients who undergo newer biologic and scaffoldbased treatments. Therefore, we aimed to assess postoperative outcomes in patients with osteochondral defects of the knee treated with BMAC and CDM and found favorable results.

The findings of this study include diminished pain and favorable functional outcomes following arthroscopic BMAC and CDM implantation in osteochondral defect patients. It is important to note that the improvement in VAS scores, UCLA, KOS-ADL, and KOS-Sports observed in this study are based on statistical significance. However, several studies have reported the minimally clinical important difference (MCID) or substantial clinical benefit (SCB) for various patientreported outcome measures to quantify the clinical significance of these values. ${ }^{27}$ According to previous studies assessing patients with cartilage defects, the MCID for VAS scores has been reported to be 2.7, the MCID for KOS-ADL was 10 (with a standard deviation (SD) of 3.7), and the SCB for KOS-ADL was 17 (SD of 3.9). ${ }^{28,29}$ Six patients in our study $(43 \%)$ demonstrated improvements in VAS scores that exceeded the MCID whereas 13 patients (93\%) demonstrated improvements KOS-ADL scores that exceeded both the MCID and the SCB. Thus, arthroscopic treatment with BMAC and CDM implantation also may have clinical significance. Although the studies that report these MCIDs are based on patients with cartilage defects, the treatments in these studies varied and included MF, mosaicplasty, OATs, and ACI/MACI. Given the novelty of interventions with biologic and scaffold-based therapies, we believe MCIDs derived from these specific treatments will emerge and better reflect their clinical implications. Despite this, correlating our results with established MCIDs provides valuable insight as to how this intervention may be affecting patients.

Given the advent of newer biologic therapies, several studies, similar to ours, have evaluated clinical outcomes in patients receiving BMAC. In their prospective cohort study, Gobbi et al. ${ }^{30}$ evaluated postoperative outcomes in patients with grade IV cartilage defects of the knee, as classified by the ICRS. The authors compared 50 patients treated with MF with 27 patients treated with HA-based scaffold and BMAC and found greater Tegner, International Knee Documentation Committee, and Knee Injury and Osteoarthritis Outcomes Score (KOOS) scores in the HA-BMAC group at 5 years. Another nonrandomized prospective trial performed by Gobbi et al. ${ }^{31}$ evaluated outcomes among patients with patellofemoral chondral lesions treated with the same HA-derived scaffold. The authors found that patients treated with BMAC $(\mathrm{n}=18)$ had greater 
Table 4. Clinical and Functional Outcomes Stratified by Osteochondral Location

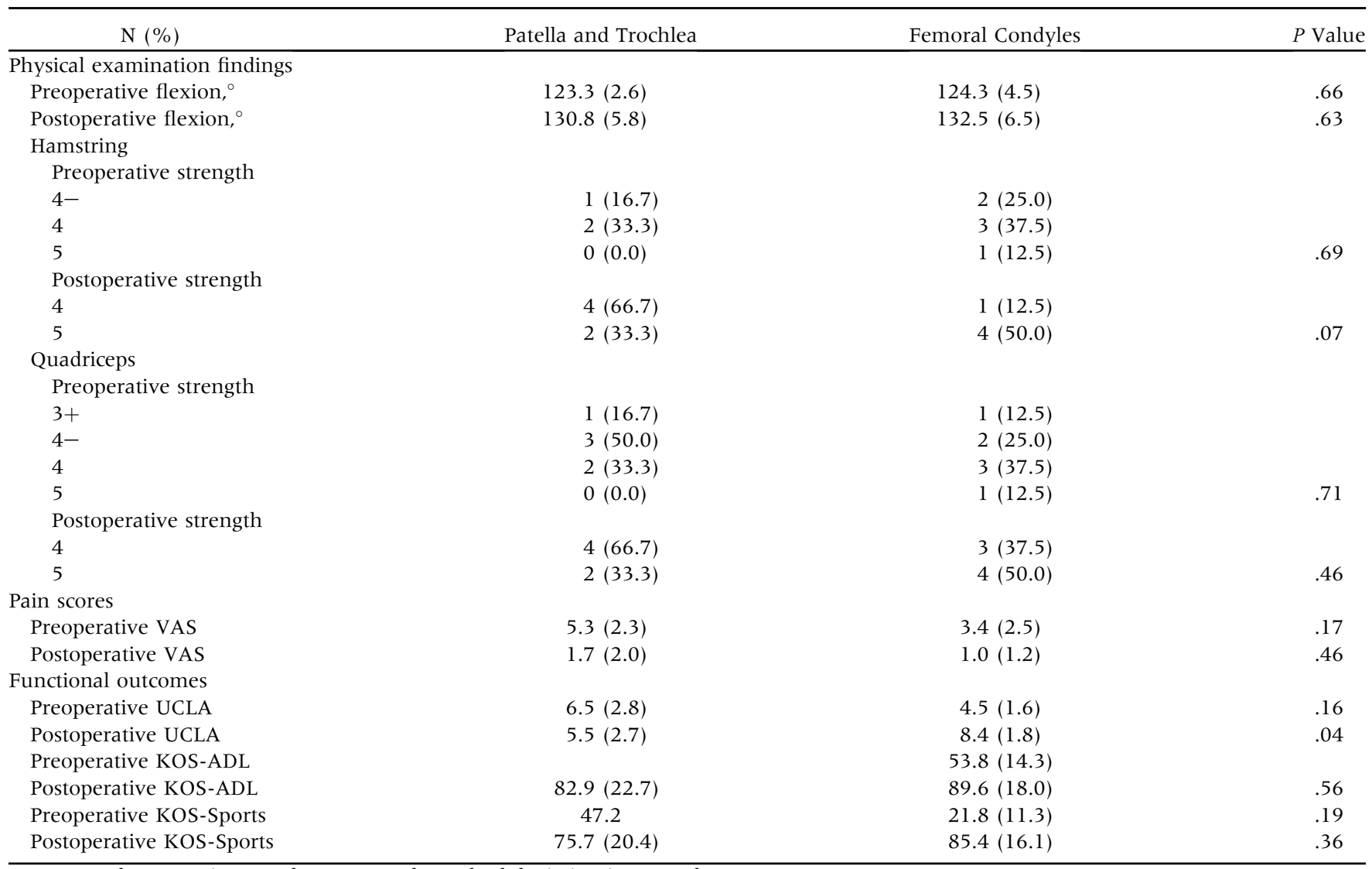

NOTE. Values are given as the mean and standard deviation in parentheses.

ADL, activities of daily living; BMAC, bone marrow aspirate concentrate; KOS, Knee Outcome Survey; UCLA: University of California, Los Angeles Activity Score; VAS, visual analog scale.

International Knee Documentation Committee subjective scores than patients treated with matrix-induced autologous chondrocyte implantation $(n=19)$ at a minimum of 3-year follow up. Similar to our study, these studies assessed patients with grade IV ICRS defects. In contrast, however, these studies performed miniarthrotomies as the approach for implantation. Although the authors concluded that BMAC is a viable

Table 5. Complications for Patients With Osteochondral Defect Treated With BMAC and CDM

\begin{tabular}{lc}
\hline \multicolumn{1}{c}{ Postoperative complications } & $\mathrm{n}(\%)$ \\
\hline Stitch abscess & $1(7.1)$ \\
Baker's cyst & $1(7.1)$ \\
Painful hardware & $1(7.1)$ \\
HA injection & $1(7.1)$ \\
Subsequent surgeries & $1(7.1)$ \\
$\quad$ Open cyst excision & $1(7.1)$ \\
Removal of hardware (two 4.5 -mm tibial & \\
$\quad$ tubercle screws) & $10.5(2.9-17.4)$ \\
Mean time to subsequent surgeries, mo & \\
\hline BMAC, bone marrow aspirate concentrate; CDM, cartilage-derived \\
matrix; HA, hyaluronic acid.
\end{tabular}

option for the treatment of osteochondral defect of the knee, our study demonstrates similar advantages of BMAC use with an arthroscopic approach albeit in the short term.

Although we describe favorable clinical outcomes, few studies report on clinical outcomes in regards to CDM treatment. Carter et al. ${ }^{32}$ published a 6-patient case series based on treatment of chondral defects of the knee with MF and BioCartilage. The authors found that KOOS scores ranged from 24 to 93 between 6 and 24 months postoperatively. Despite the variability in scores, the authors found specific findings on magnetic resonance imaging correlate with greater KOOS scores. These include the preservation of greater than 50\% thickness compared with surrounding native cartilage, mild irregularity of subchondral plate, vertical low signal intensity lines, and mild or no bone marrow edema. The BioCartilage scaffold consists of extracellular matrix that contains type II collagen, proteogylcans, and growth factors. ${ }^{32}$ In contrast to MF, which primarily produces fibrocartilage ${ }^{33}$ with type-1 collagen, the BioCartilage construct is, in theory, intended to promote regeneration of hyaline-like 
cartilage. Given that BMAC and CDM implantation do not lead to donor-site morbidity, require one stage, are less costly, and can be performed through minimally invasive techniques, we believe this may serve as an alternative to conventional treatment (i.e., MF, OATs, ACI, and matrix-induced autologous chondrocyte implantation).

\section{Limitations}

This study has several limitations. Due to the retrospective design, it is difficult to establish cause and effect between the intervention and treatment success. The sample size was small, which increased the possibility of a type II error. In addition, this study lacks a control group and longer follow-up, which further limits the scope of our results. One patient received a series of HA injections postoperatively and interventions such as these can influence the results. In addition, the composition of the BMAC preparation was not assessed. BMAC preparation is not standardized in the literature, and this can explain some of the differences found on the efficacy of this treatment. ${ }^{34}$ Finally, all patients underwent at least one additional procedure, which creates a very heterogenous study group.

\section{Conclusions}

This study demonstrated arthroscopic BMAC and CDM implantation appear to be safe and have the potential to improve patient outcomes in the short-term postoperative period.

\section{References}

1. Hjelle K, Solheim E, Strand T, Muri R, Brittberg M. Articular cartilage defects in 1,000 knee arthroscopies. Arthroscopy 2002;18:730-734.

2. Widuchowski W, Widuchowski J, Trzaska T. Articular cartilage defects: Study of 25,124 knee arthroscopies. Knee 2007;14:177-182.

3. D'Ambrosi R, Valli F, De Luca P, Ursino N, Usuelli F. MaioRegen osteochondral substitute for the treatment of knee defects: A systematic review of the literature. J Clin Med 2019;8:783.

4. Buckwalter JA. Articular cartilage: injuries and potential for healing. J Orthop Sports Phys Ther 1998;28:192-202.

5. Scillia AJ, Aune KT, Andrachuk JS, et al. Return to play after chondroplasty of the knee in national football league athletes. Am J Sports Med 2015;43:663-668.

6. Ackermann J, Mestriner AB, Shah N, Gomoll AH. Effect of autogenous bone marrow aspirate treatment on magnetic resonance imaging integration of osteochondral allografts in the knee: A matched comparative imaging analysis. Arthroscopy 2019;35:2436-2444.

7. Perera JR, Gikas PD, Bentley G. The present state of treatments for articular cartilage defects in the knee. Ann $R$ Coll Surg Engl 2012;94:381-387.

8. Rath B, Eschweiler J, Betsch M, Gruber G. Cartilage repair of the knee joint. Orthopade 2017;46:919-927 [in German].
9. Everhart JS, Campbell AB, Abouljoud MM, Kirven JC, Flanigan DC. Cost-efficacy of knee cartilage defect treatments in the United States. Am J Sports Med 2020;48: 242-251.

10. Stevens HY, Shockley BE, Willett NJ, et al. Particulated juvenile articular cartilage implantation in the knee: A 3year EPIC- $\mu \mathrm{CT}$ and histological examination. Cartilage 2014;5:74-77.

11. Widuchowski W, Lukasik P, Kwiatkowski G, et al. Isolated full thickness chondral injuries. Prevalence and outcome of treatment. A retrospective study of 5233 knee arthroscopies. Acta Chir Orthop Traumatol Cech 2008;75: 382-386.

12. Rizzello G, Longo UG, Petrillo S, et al. Growth factors and stem cells for the management of anterior cruciate ligament tears. Open Orthop J 2012;6:525-530.

13. Fortier LA, Potter HG, Rickey EJ, et al. Concentrated bone marrow aspirate improves full-thickness cartilage repair compared with microfracture in the equine model. J Bone Joint Surg Am 2010;92:1927-1937.

14. Ivkovic A, Pascher A, Hudetz D, et al. Articular cartilage repair by genetically modified bone marrow aspirate in sheep. Gene Ther 2010;17:779-789.

15. Kon E, Roffi A, Filardo G, Tesei G, Marcacci M. Scaffoldbased cartilage treatments: With or without cells? A systematic review of preclinical and clinical evidence. Arthroscopy 2015;31:767-775.

16. Manfredini M, Zerbinati F, Gildone A, Faccini R. Autologous chondrocyte implantation: a comparison between an open periosteal-covered and an arthroscopic matrixguided technique. Acta Orthop Belg 2007;73:207-218.

17. Ferruzzi A, Buda R, Faldini C, et al. Autologous chondrocyte implantation in the knee joint: Open compared with arthroscopic technique. Comparison at a minimum follow-up of five years. J Bone Joint Surg A 2008:90-101.

18. Zeifang F, Oberle D, Nierhoff C, Richter W, Moradi B, Schmitt H. Autologous chondrocyte implantation using the original periosteum-cover technique versus matrixassociated autologous chondrocyte implantation: A randomized clinical trial. Am J Sports Med 2010;38:924-933.

19. Chadha N, Dang A, Sampson E, et al. Porous cartilagederived matrix scaffolds for repair of articular cartilage defects. Poster 0735. Procs ORS 2012, San Francisco, California.

20. Case JM, Scopp JM. Treatment of articular cartilage defects of the knee with microfracture and enhanced microfracture techniques. Sports Med Arthrosc 2016;24: 63-68.

21. Goyal D, Keyhani S, Lee EH, Hui JHP. Evidence-based status of microfracture technique: A systematic review of Level I and II studies. Arthroscopy 2013;29:1579-1588.

22. Andrade R, Vasta S, Pereira R, et al. Knee donor-site morbidity after mosaicplasty - a systematic review. J Exp Orthop 2016;3.

23. Derrett S, Stokes EA, James M, Bartlett W, Bentley G. Cost and health status analysis after autologous chondrocyte implantation and mosaicplasty: A retrospective comparison. Int J Technol Assess Health Care 2005;21: 359-367.

24. de Windt TS, Sorel JC, Vonk LA, Kip MMA, Ijzerman MJ, Saris DBF. Early health economic modelling of 
single-stage cartilage repair. Guiding implementation of technologies in regenerative medicine. J Tissue Eng Regen Med 2017;11:2950-2959.

25. Dávila Castrodad IM, Mease SJ, Werheim E, McInerney VK, Scillia AJ. Arthroscopic chondral defect repair with extracellular matrix scaffold and bone marrow aspirate concentrate. Arthrosc Tech 2020;9:e1241-el247.

26. Dwyer T, Martin CR, Kendra R, et al. Reliability and validity of the arthroscopic international cartilage repair society classification system: Correlation with histological assessment of depth. Arthroscopy 2017;33:1219-1224.

27. Harris JD, Brand JC, Cote MP, Faucett SC, Dhawan A. Research pearls: The significance of statistics and perils of pooling. Part 1: Clinical versus statistical significance. Arthroscopy 2017;33:1102-1112.

28. Wang D, Chang B, Coxe FR, et al. Clinically meaningful improvement after treatment of cartilage defects of the knee with osteochondral grafts. Am J Sports Med 2019;47:71-81.

29. Jones KJ, Kelley BV, Arshi A, McAllister DR, Fabricant PD. Comparative effectiveness of cartilage repair with respect to the minimal clinically important difference. Am J Sports Med 2019;47:3284-3293.
30. Gobbi A, Whyte GP. One-stage cartilage repair using a hyaluronic acid-based scaffold with activated bone marrow-derived mesenchymal stem cells compared with microfracture. Am J Sports Med 2016;44:2846-2854.

31. Gobbi A, Chaurasia S, Karnatzikos G, Nakamura N. Matrix-induced autologous chondrocyte implantation versus multipotent stem cells for the treatment of large patellofemoral chondral lesions: A nonrandomized prospective trial. Cartilage 2015;6:82-97.

32. Carter AH, Guttierez N, Subhawong TK, et al. MR imaging of BioCartilage augmented microfracture surgery utilizing 2D MOCART and KOOS scores. J Clin Orthop Trauma 2018;9:146-152.

33. Pipino G, Risitano S, Alviano F, et al. Microfractures and hydrogel scaffolds in the treatment of osteochondral knee defects: A clinical and histological evaluation. J Clin Orthop Trauma 2019;10:67-75.

34. Piuzzi NS, Hussain ZB, Chahla J, et al. Variability in the preparation, reporting, and use of bone marrow aspirate concentrate in musculoskeletal disorders: A systematic review of the clinical orthopaedic literature. J Bone Joint Surg Am 2018;100:517-525. 